\title{
DIFFERENTIAL ROTATION IN THE SOLAR INTERIOR
}

\author{
MAURICE J. CLEMENT \\ David Dunlap Observatory, University of Toronto, Canada
}

\section{Introduction}

One of the big problems in stellar rotation which has been the object of much debate recently concerns the magnitude of the angular velocity in the central regions of the sun. It is a good example of our general ignorance of the distribution of angular momentum in the interiors of stars. There is good reason, of course, for this ignorance. One can't make any direct observations and from a theoretical point of view there are many real problems such as the lack of a good theory of convection and meridian circulation, and our ignorance of the structure and magnitude of magnetic fields in the deep stellar interior. These problems among others make it very difficult, for example, to specify a surface condition on the angular velocity. It was pointed out recently (Clement, 1969; this paper is referred to hereinafter as Paper I) that such a condition might enable us to estimate the magnitude of the interior stellar rotation.

In this paper, an equilibrium model for the distribution of angular velocity in the sun is presented. The model has a surface distribution of velocity which is the same as that of the sun (by assumption) and also differs significantly in its interior properties from the model of Roxburgh (1964) for which the angular velocity is constant on spheres. In view of Roxburgh's review paper in this volume, one might ask: Why look for equilibrium distributions of velocity when we know they are likely to be unstable? It is possible that an equilibrium distribution is related in some way with the actual, time-dependent distribution. Also, there still appears to be some doubt as to the stability of distributions which differ from those with cylindrical symmetry by only a small amount. The equilibrium distributions for upper-main-sequence stars which are presented in Paper I do have approximate cylindrical symmetry with no meridian motions and so their stability or instability is, in fact, open to some question.

\section{Formulation}

To illustrate how this problem is solved it is necessary to show some of the basic equations. The mathematical formulation and notation is essentially that of Schwarzschild (1947) and Roxburgh (1964). Slow rotation is assumed and the dimensionless physical quantities are written as the sum of a spherically symmetric quantity with subscript $u$ and an axisymmetric quantity with subscript $d$ and coefficient $\lambda$ which is proportional to the square of the angular velocity at the pole. Thus,

$$
x=\frac{r}{R_{u}}, \quad \lambda=\frac{\Omega_{p}^{2} R_{u}^{3}}{G M},
$$




$$
\begin{aligned}
& p={ }_{G M^{2}}^{4 \pi R_{u}^{4}} P=p_{u}+\lambda \stackrel{{ }^{p}}{t_{u}} p_{d}, \\
& t=\frac{k}{\mu H}{ }_{G M}^{R_{u}} T=t_{u}+\lambda t_{d}, \\
& \phi={ }_{G M}^{R_{u}} \Phi=\phi_{u}+\lambda \phi_{d}, \\
& l=\frac{4 \pi r^{2}}{L_{u}} F_{r}=l_{u}+\lambda \frac{x t_{u}^{b}}{C p_{u}^{e}} l_{d},
\end{aligned}
$$

where $P, T, M$, etc. have their usual meaning. The parameters $b$ and $e$ are associated with the assumed opacity law:

$$
\kappa=\kappa_{0} \varrho^{e-1} T^{-s} \text { with } \quad b=s+e+3 .
$$

The axisymmetric quantities are expanded in a series of Legendre polynomials in the standard way; e.g.

$$
p_{d}=\sum_{j} p_{j}(x) P_{2 j}(\cos \theta)
$$

Substitution of the foregoing expressions into the standard equations of stellar structure and the elimination of the angular velocity from the equation of hydrostatic equilibrium (cf. Paper I) yields the following equations which are to be applied to the radiative core:

$$
\begin{aligned}
& x \frac{\mathrm{d} p_{j}}{\mathrm{~d} x}=Q_{j}+q_{j}-\left(2_{j}+1\right)\left(p_{j}-\phi_{j}\right)+U_{u} p_{j}-V_{u} t_{j}, \\
& x \frac{\mathrm{d} t_{j}}{\mathrm{~d} x}=U_{u}\left(e p_{j}-b t_{j}\right)-l_{j}, \\
& x \frac{\mathrm{d} \phi_{j}}{\mathrm{~d} x}=q_{j}, \\
& x \frac{\mathrm{d} q_{j}}{\mathrm{~d} x}=-q_{j}+2 j(2 j+1) \phi_{j}+W_{u}\left(t_{j}-p_{j}\right), \\
& \mathrm{d} l_{j}=-X_{u} l_{j}-2 j(2 j+1) t_{j}-Y_{u}\left[2 p_{j}+(v-2) t_{j}\right],
\end{aligned}
$$

where

$$
Q_{j}=\frac{(2 j-1)(4 j+1)}{2 j(4 j-3)}\left[(4 j-3)\left(p_{j-1}-\phi_{j-1}\right)-Q_{j-1}\right]
$$

$U_{u}, V_{u}, W_{u}$, and $X_{u}$ are as given in Paper I, and

$$
Y_{u}=\left(\frac{\mathrm{d} \ln l_{u}}{\mathrm{~d} \ln x}\right)\left(\frac{\mathrm{d} \ln t_{u}}{\mathrm{~d} \ln x}\right)=\left(\frac{\mathrm{d} \ln l_{u}}{\mathrm{~d} \ln x}\right) U_{u} .
$$


This last quantity makes allowance for the nuclear energy generation in the radiative core. In Equation (12), $v$ is the temperature exponent of the energy generation law.

The foregoing equations point out the source of difficulty inherent in this problem of differential rotation. Equations (8)-(12) are homogeneous except for the quantity $Q_{j}(x)$ which clearly couples equations of different Legendre orders. As the problem now stands, $Q_{0}$ (say) is undefined. Given a function $Q_{0}(x)$, all the equations (including the relatively simple ones for the convective envelope) could be integrated in a straightforward way. And if there were no conditions to satisfy other than the standard boundary conditions, then $Q_{0}$ would be quite arbitrary. Thus, an unlimited number of velocity distributions could be generated by choosing different $Q_{0}$. For example, $Q_{0}$ can be chosen in quite a simple way such that the angular velocity is constant on spheres. This is essentially how Roxburgh obtained his model. But solutions found in this way are mathematical ones and have little or no physical basis. One should choose a $Q_{0}$ which gives a velocity distribution satisfying some justifiable boundary condition. This has been done for the upper-main-sequence stars (cf. Paper I). The particular condition applied to these stars will not be discussed here because it has no relevance for lower-main-sequence stars which have convective envelopes. However, in the case of the sun, there is an obvious boundary condition which is just the observed distribution of angular velocity on the surface; viz. (cf. Allen, 1963)

$$
\Omega / \Omega_{p}=1.0000+0.2312 \sin ^{2} \theta .
$$

What has been done is to determine that unique $Q_{0}(x)$ which gives this surface distribution.

\section{Assumptions}

A number of assumptions have been made; some of these are obvious from the foregoing equations.

(i) The only effective forces are those of pressure, gravitation, and rotation: This means that there are no acceleration forces due to a solar-wind torque or to meridian circulation; i.e., there is steady state and no motions other than pure rotation. This assumption also means that there are no viscous or magnetic stresses. It is a purely hydrodynamic model and if it turns out that the magnetic field in the deep interior of the sun is very strong then the model presented here may have to be modified.

(ii) Uniform chemical composition: A gradient in the mean molecular weight may affect the equilibrium distribution of angular velocity; it has already been shown to be important for stability. This problem will be examined at another time.

(iii) Adiabatic convective envelope: The effect of this assumption is to make the angular velocity in the envelope constant on cylinders and so the velocity distribution at the bottom of the convective region is directly related in an obvious way to the observed distribution at the surface. We know that the temperature gradient near the surface is actually superadiabatic and what effect this will have on the velocity distribution at the bottom of the envelope is not clear. However, numerical experi- 
ments show that the angular velocity at the center of the sun depends only weakly on the actual distribution at the bottom of the convective envelope. So unless viscous stresses, meridian circulation, and/or a superadiabatic temperature gradient drastically affect the rotation law in the envelope, the assumption that these effects are negligible will not affect the model's central rotation rate in a significant way.

\section{Results}

As already indicated, the object of the present problem is to find that particular $Q_{0}(x)$ which gives the observed solar velocity distribution. This is essentially a matter of curve-fitting and to this end $Q_{0}$ is written as a polynomial in $x^{2}$ (there can be no linear term; otherwise, the pressure gradient at the origin would differ from zero as can be easily verified). The polynomial coefficients are determined by matching the various physical quantities in the radiative core with those in the convective envelope. The number of terms $J+1$ (say) in the expansion for $Q_{0}$ must equal the number of Legendre orders to be included in the analysis. So far, terms up to and including $P_{10}(\cos \theta)$ have been considered and this means terms up to $x^{10}$ for $Q_{0}(x)$ (i.e., up to $J=5$ ). By considering solutions with a smaller number of terms, one can, of course, see how the model converges. In fact, $Q_{0}(x)$ converges quite well because the curves corresponding to $J=3,4$, and 5 are almost indistinguishable on the scale shown in Figure 1 (for clarity, only one curve has been drawn). That is, the model described by terms up to only order $P_{6}(\cos \theta)$ is almost as accurate as one including terms up to $P_{10}(\cos \theta)$ as far as $Q_{0}$ is concerned. Unfortunately, the central angular velocity depends not on

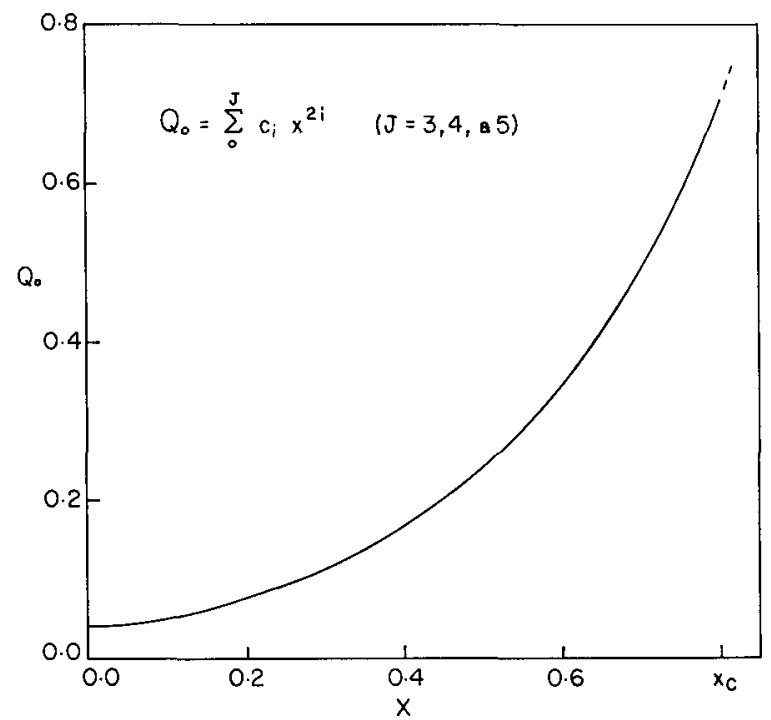

Fig. 1. The function $Q_{0}(x)$ for a solar model in which the radiative core extends out to $x_{\mathrm{c}}=0.80$. The same quantity can be found in the convective envelope with the aid of Equation (8) but there it is determined mainly by the assumed velocity distribution on the surface. 
$Q_{0}$ but on the second derivative of $Q_{0}$ at the origin. As with all curve-fitting problems, a polynomial with a finite number of terms can't represent the higher derivatives of a function as accurately as the function itself. Consequently, the central angular velocity is not as well determined as the convergence of $Q_{0}$ would indicate.

Figure 2 illustrates the velocity distribution corresponding to the $Q_{0}(x)$ for $J=5$. The central angular velocity is $40 \%$ higher than that at the pole and less than $15 \%$ higher than the equatorial value. This relatively low angular velocity does not change significantly if the envelope is made to rotate rigidly or if the opacity and nuclearenergy-generation laws are changed. It is evident from the figure that the distribution of velocity has no cylindrical symmetry in the radiative core and is therefore likely to be unstable in view of the analysis of Goldreich and Schubert (1967). If there really are no stable equilibrium velocity distributions then one might wonder how the timeaverage of the actual velocity compares with the equilibrium distribution. The velocity at different points may oscillate about the equilibrium values in which case the latter would be representative of the actual distribution. The resolution of this problem must await future investigations.

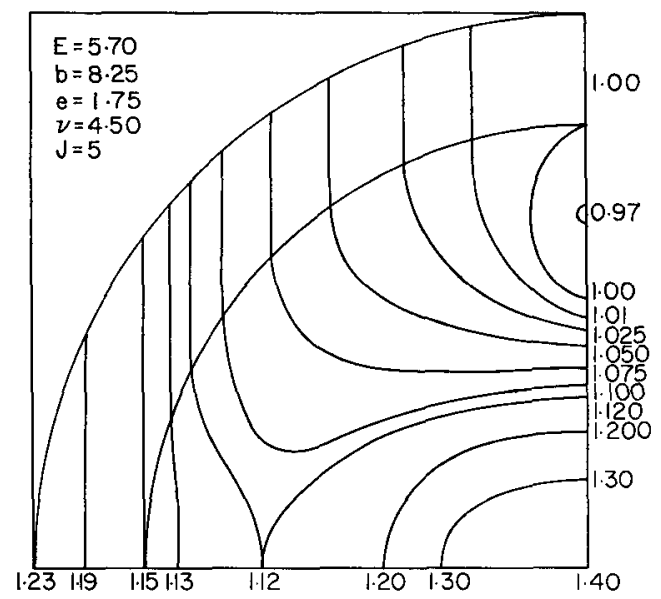

Fig. 2. The curves of constant angular velocity $\left(\Omega / \Omega_{\mathrm{p}}\right)$ in a quadrant of a meridian plane. The equator is at the bottom left and the pole at the top right. The region between the two quarter-circles is the convective envelope which is governed by the Schwarzschild parameter $E=5.70$. The other parameters characterizing this model are shown at the upper left of the figure (cf. text for definitions).

\section{References}

Allen, C. W.: 1963, Astrophysical Quantities, 2nd ed., University of London, Athlone Press, p. 179. Clement, M. J.: 1969, Astrophys. J. 156, 1051.

Goldreich, P. and Schubert, G.: 1967, Astrophys. J. 150, 571.

Roxburgh, I. W.: 1964, Monthly Notices Roy. Astron. Soc. 128, 157.

Schwarzschild, M.: 1947, Astrophys. J. 106, 427.

\section{Discussion}

Livingston: The models indicate a definite change of angular velocity (differential rotation) right to 
the solar equator. I will just comment that, observationally, there is no differential motion seen through the sunspot zone. At least this seems to be the case spectroscopically, and at this part of the solar cycle.

Roxburgh: I would be surprised if you got approximately cylindrical rotation. Since we know there are no equilibrium solutions in which this is the case and one would expect that the departures from cylindrical symmetry are as big as the rotation itself.

Clement: This did not turn out to be the case for the upper main-sequence models for which the distribution of angular velocity is approximately constant on cylinders. In fact, in the limit of slow rotation, the departures from cylindrical symmetry are independent of the magnitude of the angular velocity and depend only on the mass, chemical composition, and boundary conditions. 